\section{Particle beam interface}

\section{B. Güssregen}

Merck KGaA, Darmstadt, Deutschland
Beschreibung Das „particle beam interface“ erlaubt die Kopplung von $>$ Flüssigkeitschromatographie und $>$ Massenspektrometrie. Die mobile Phase wird durch Heliumgas entfernt, die Ionisierung des Analyten erfolgt durch Elektronenstoßionisation (EI) oder chemische Ionisation (CI). „Particle beam interface" wird heute meist durch Elektrospray (ESI) oder „atmospheric pressure chemical ionisation“ (APCI) verdrängt. 